\title{
Multiple Cavitating Pulmonary Nodules Secondary To Hodgkin's Lymphoma
}

\author{
Abdul Azih, MN, ${ }^{\mathrm{a}}$ Rahman, AA, ${ }^{\mathrm{b}} \mathrm{Hin}, \mathrm{HS},{ }^{\mathrm{a}}$ Chunn, $\mathrm{KY},{ }^{\mathrm{c}}$ Kori $\mathrm{AN}^{\mathrm{a}}$ \\ ${ }^{a}$ Respiratory unit, Department of Internal Medicine, Kulliyyah of Medicine, International Islamic University \\ Malaysia, Kuantan Pahang \\ ${ }^{\mathrm{b}}$ Department of Pathology, Hospital Tengku Ampuan Afzan, Kuantan, Pahang \\ 'Hematology unit, Department of Internal Medicine, Tengku Ampuan Afzan, Kuantan, Pahang
}

\begin{abstract}
We report a 26-year old lady who presented with chronic cough and breathlessness associated with subtle TB symptoms for 1 year. Her CT thorax showed multiple cavitating pulmonary nodules with mediastinal and cervical lymphadenopathy. Cervical lymph node biopsy and CT-guided pulmonary biopsy at our centre confirmed the diagnosis of Hodgkin's lymphoma with pulmonary infiltrations. She was successfully treated with $A B V D$ regime but later developed life-threatening bleomycin-induced pulmonary fibrosis. Sadly, she succumbed to respiratory failure due to severe pneumonia with possibility of bleomycin-induced pulmonary fibrosis. Multiple cavitating pulmonary nodules secondary to lymphoma is rare and in TB endemic area, it may result in delayed diagnosis and treatment.
\end{abstract}

Keywords: Multiple cavitating pulmonary nodules, Hodgkin's lymphoma, bleomycin, bleomycin-induced lung injury

\section{INTRODUCTION}

Pulmonary and mediastinal involvements in lymphoma are relatively common. However, multiple cavitating pulmonary nodules in both Hodgkin's and non-Hodgkin's lymphoma are extremely rare. Only few of such cases are reported. In Hodgkin's disease, painless localized peripheral mediastinal lymphadenopathy is the commonest presentation followed by mediastinal mass. In TB endemic region, atypical presentations may lead to late diagnosis.

\section{Case presentation}

Ms. WS, a 26 year-old single teacher, initially presented in April 2012 to Hospital Sultanah Nur Zahirah Kuala Terengganu, Malaysia with chronic respiratory symptoms. She complained of cough, blood-streak sputum, breathlessness and intermittent fever for 1 month. She also had weight loss of 20 kilogram within the year. After her initial chest radiograph that showed multiple ill-defined nodules and cavities with negative tuberculosis (TB) work-up, a first CT thorax was arranged at this the centre. It showed multiple cavitating

Corresponding author:

Dr. Muhammad Naimuddin Abdul Azih,

Respiratory unit,

Department of Internal Medicine

Kulliyyah of Medicine,

International Islamic University Malaysia

Kuantan Pahang

Email: naimedicine@hotmail.com pulmonary nodules with mediastinal and cervical lymphadenopathies (Figures 1 \& 2 ). Her first cervical lymph node biopsy in what year was interpreted as Kikuchi's disease. She was later treated empirically as smear-negative pulmonary tuberculosis with no improvement. However she refused further invasive investigations and was treated empirically with antifungal drug and later with oral corticosteroid by the treating physician.

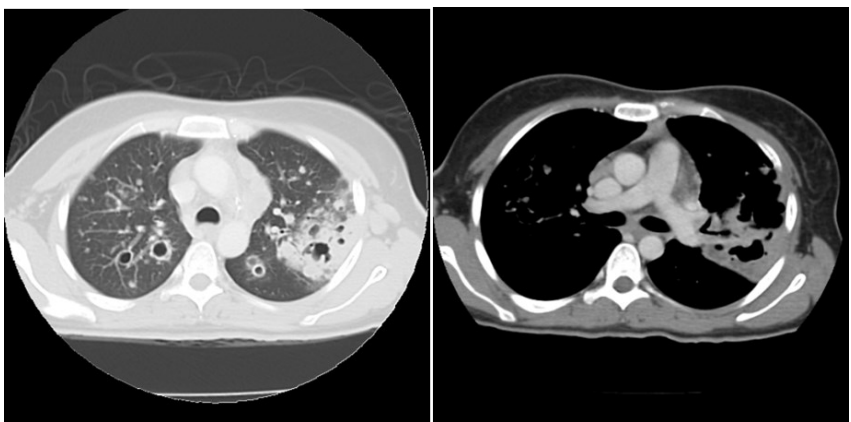

Figure 1 \& 2 CECT thorax done on 14/10/12 showed multiple cavitating pulmonary nodules with multiple mediastinal lymphadenopathy. Consolidation of left lingular segment is also seen.

A year later in August 2013, due to her worsening condition, she was referred to our centre for second opinion. During admission, she appeared cachectic and breathless at rest. Her chest radiograph showed multiple nodular pulmonary lesions and ill-defined cavitations. Her repeated CT scan of neck, thorax, abdomen and pelvis showed worsening pulmonary lesions, multiple cervical, mediastinal, abdominopelvic lymphadenopathy with matted inguinal lymph nodes. A second cervical lymph node biopsy was done at our centre in (what month, year) and it 
showed a typical feature of Hodgkin's lymphoma (Figures 3 \& 4). Few days after admission, she developed type 2 respiratory failure and she was intubated due to respiratory failure secondary to severe pneumonia and she was transferred to intensive care unit. A CT-guided pulmonary biopsy was carried out and the histopathological examination was consistent with that of cervical lymph node biopsy.

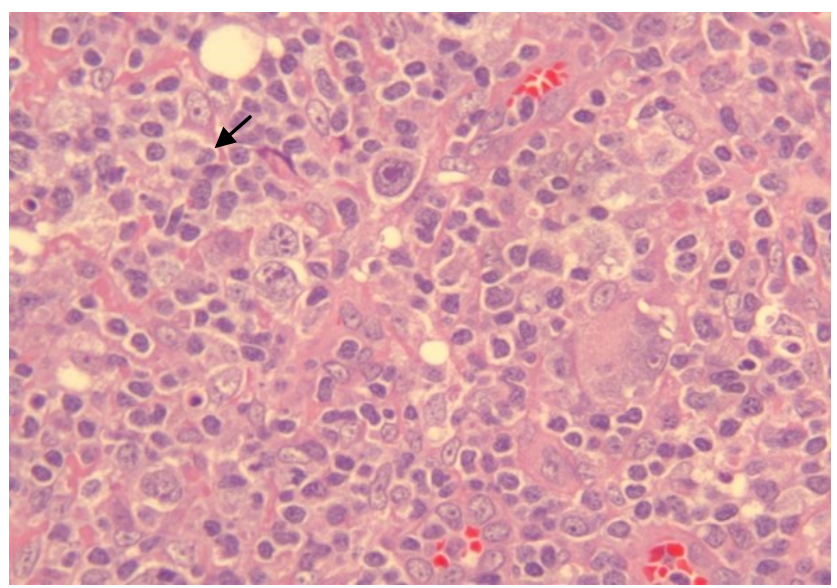

Figure 3: Histopathological examination of cervical lymph node. The arrow is showing typical Hodgkin's cell (Reed-Sternberg cell) which is large and has prominent eosinophilic nucleoli There are amphophilic cytoplasms. There are many inflammatory cells in the background including lymphocytes, plasma cells and occasional eosinophils

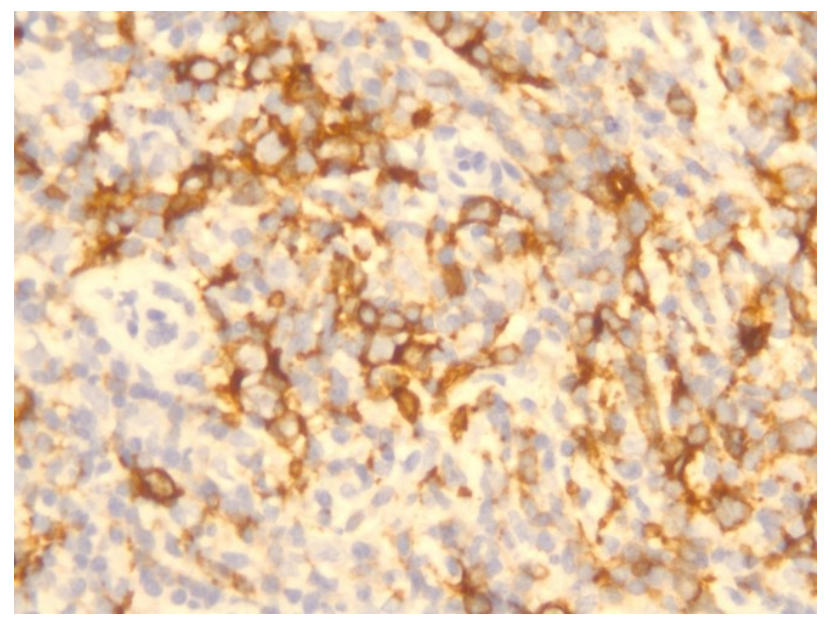

Figure 4: The immunohistochemistry of lung nodules showed positivity for CD 30 shows similar findings as in the lymph node lesion.

Therefore she was diagnosed as stage 4 Hodgkin's lymphoma with pulmonary infiltrations and was referred to clinical haematologist. The ABVD regime that consists of adriamycin, bleomycin, vincristine and dacarbazine was started after infectious parameters were under control. She responded well albeit prolonged intubation due to ventilator associated pneumonia and secondary pneumothorax. She was extubated after 3 weeks of mechanical ventilation and weaned off gradually with tracheostomy that was successfully closed later. After completion of chemotherapy, she was well and able to attend our respiratory clinic follow-up without oxygen and ambulate independently with minimal exertional breathlessness. Her repeated CT thorax showed complete resolution of the cavitating pulmonary nodules with residual large bullae bilaterally and mild pulmonary fibrosis. She remained in remission for 1 month.

Unfortunately, after 1 month of completing chemotherapy, she developed worsening breathlessness. After a negative extensive work-up for infections and other possible causes including relapse of the underlying Hodgkin's lymphoma, she was diagnosed with severe pneumonia possibly due to bleomycin-induced pulmonary fibrosis. She was admitted and prescribed with broadspectrum antibiotics and empirical treatment for pneumocystis pneumonia. After Following a multidisciplinary discussion, a detailed discussion with her family members to embark on for a therapeutic trial of Imatinib Mesylate after she failed to respond to antimicrobial therapy and high-dose corticosteroids. However, she did not respond to the treatment and later succumbed to respiratory failure in the intensive care unit after two weeks on mechanical ventilation.

\section{Discussion}

Hodgkin's lymphoma $(\mathrm{HL})$ is a rare cause of multiple cavitating pulmonary nodules where the exact prevalence and incidence is unknown since only a few cases were reported. ${ }^{1}$ Most patients with classical HL present with painless localized peripheral lymphadenopathy, typically involving the cervical region. ${ }^{1}$ Aissaoiu et al. mentioned that in 1991, only 53 reported cases of cavitating lymphoma were reported in which part of the world/region. ${ }^{1}$ in $1991 .{ }^{1}$ Jackson et al. reported in 1994, 2 cases of similar presentation in NHL. ${ }^{2}$ The radiological patterns of pulmonary parenchymal involvement in lymphoma were described by $\mathrm{N}$. Berkman et al (1993). According to Berkman et al, pulmonary nodules are the most frequent manifestation of lymphoma with parenchymal involvement.

These nodules tend to be small, round or oval in shapes and with varying sizes. However, much larger masses involving an the entire lobe have been reported. ${ }^{3}$ The nodules tend to have ill-defined margins and the margin can be speculated or associated with satellite nodules around them ${ }^{4}$. They are usually less rounded and regular compared to pulmonary metastases. ${ }^{4}$ Air bronchograms may be seen traversing these nodules. As seen in this patient, cavitations with a thin or thick surrounding wall may occur. Occasionally it is associated with air-fluid level. ${ }^{4}$ Patients can have a single or multiple, unilateral or bilateral nodules. ${ }^{4}$ Geographically, it affects the lower lobes more commonly than the upper lobes. ${ }^{4}$ Showers of nodules are uncommon but have been described in end stage disease. ${ }^{4}$ The other interesting feature in of $\mathrm{HL}$ is that, the nodules frequently traverse the lobar fissures as seen in this patient. In $\mathrm{HL}$, the pulmonary nodules can be 
located subpleurally as plaque-like lesions that may resemble pleural thickening or pulmonary infarcts. ${ }^{4}$ Apart from pulmonary nodules, the other known radiological patterns of pulmonary parenchymal involvement in lymphoma are bonchovascular-lymphangitic pattern and pneumonic -alveolar patterns. ${ }^{4}$

Generally, the causes of cavitating pulmonary nodules can be divided into infectious and noninfectious aetiology. In this patient, infectious cause was the initial working diagnosis since tuberculosis is an endemic infection in Malaysia. However, high index of suspicion is paramount if work-ups for tuberculosis are negative and further investigations are warranted to exclude other rare causes of cavitating pulmonary nodules including lymphoma. The common differential diagnoses of cavitating pulmonary nodules with their characteristic features are as follows. ${ }^{5}$

Disease Condition

Characteristic Features

Noninfectious etiology

Granulomatosis with

polyangitis

Hematogenous metastases

Embolism

Lymphomatoid granulomatosis

Langerhans cell histiocytosis

Primary bronchogenic

carcinoma

Lymphoma

Sarcoidosis
Nodules and infiltrates are frequently seen. Sinonasal involvement commonly associated. Nodules may show cavitation in nearly one-half the cases. No lobar predilection.

Lower lobe predominance, feeding vessels frequently seen.

Infarction and subsequent necrosis may lead to appearance of a cavitary lesion. Usually peripheral without any specific lobar predilection.

Basal predominant involvement may be confused with pulmonary abscess.

Thin-walled cysts and cavities are usually seen. Thicker-walled cavities can also be observed. Sparing of the basal areas is usually seen.

Cavitation more frequent in squamous cell carcinoma; multiple cavitating nodules are uncommon.

Cavitation more frequent in individuals infected with HIV.

Cavitary lesions seen in advanced fibrotic stages of the disease with an upper lobe distribution. Accompanying perilymphatic nodules and/or mediastinal lymphadenopathy may be seen.

\section{Disease Condition}

\section{Characteristic Features}

\section{Infectious etiology}

\begin{tabular}{ll}
\hline Septic emboli & $\begin{array}{l}\text { Peripheral/subpleural, more likely lower lobe predominant. Typically, found in } \\
\text { varying stages of cavitation with some non-cavitary nodules. }\end{array}$ \\
Tuberculosis & Upper lobes/apices or superior segments of lower lobe predominantly involved. \\
Echinococcosis & $\begin{array}{l}\text { Usually seen as homogenous rounded pulmonary nodule/masses. Rupture can lead to } \\
\text { appearance of cavitating nodule. Surrounding pulmonary is usually normal. }\end{array}$ \\
\hline
\end{tabular}

\section{Conclusion}

Lymphoma is a rare cause of multiple cavitating pulmonary nodules. It can be a manifestation of primary pulmonary lymphoma or due to lymphomatous pulmonary infiltrations as seen in this case. High index of suspicion followed by a lung biopsy is necessary to diagnose the disease early.

\section{REFERENCES}

1. Aissaoui A, Achour $\mathrm{H}$, Hamlaoui $\mathrm{H}$, Khellaf $\mathrm{M}$. Hodgkin's disease of the pulmonary with cavitation and apparently primary. Apropos of a case. Cancer. 1991 Sep 1;68(5):930-3.

2. A. Jackson a, K.T. Tung a, 1, G.M. Mead, Multiple cavitating lesions in non-Hodgkin lymphoma. Clinical Radiology (1994) 49, 883885.
3. N. Berkman and R. Breuer, Institute of Pulmonology, Pulmonary involvement in lymphoma. Hadassah University Hospital, Jerusalem, Israel Respiratory Medicine (1993) 87,85-92.

4. Fraser RG, Pare JAP, Pare PD, Fraser R, Genereaux GP. Diagnosis of diseases of the chest 3rd ed. Philadelphia: WB Saunders Co. 1989: 1507-1554.

5. Karan Madan, MD, DMa, Suvendu Purkait, MDa, Sudheer Arava, MDa, Ashu S. Bhalla, MDa, Rakesh Kumar, MDa, Randeep Guleria, MD, DMa, A 79-Year-Old Woman With Bilateral Cavitating Pulmonary Nodules. Chest, Volume 145, Issue 6, June 2014, Pages 1419-1424. 